\title{
Variance-Time Curve for Packet Streams Generated by Exponentially Distributed ON/OFF Sources
}

\author{
Georgios Y. Lazarou and Victor S. Frost
}

\begin{abstract}
In this letter we provide a solution to an open problem in network traffic characterization. Specifically we present a closed-form expression of the variance-time curve for a packet stream generated by exponentially distributed ON/OFF sources. So far, the variance-time curve for such processes was obtained by numerical analysis at the desired time scales. We also show that under low and medium loads, the variance-time curve obtained by approximating the ON/OFF/Exponential source as a stationary fluid source is over-estimated. Lastly, as a by-product of our analysis, we present a new mathematical identity based on the incomplete Gamma function.
\end{abstract}

Index Terms-Traffic characterization, variance-time curve, ON/OFF/exponential source.

\section{INTRODUCTION}

$\mathbf{T}$ RAFFIC is the driving force behind all telecommunication activities, and models are of crucial importance for evaluating network performance. In this letter we consider a traditional model, namely the ON/OFF Exponential traffic source model. This traffic model became popular when it was used in [1] to characterize the aggregate packet arrival process generated by the superposition of separate voice streams. For computing the index of dispersion for counts (IDC) for an aggregated voice-packet stream, the variance-time curve was obtained in [2] by numerical analysis at the desired time scales.

Our main goal in this letter is to derive a closed-form expression of the variance-time curve for the packet streams generated by such traffic sources. This closed-form expression of the variance-time is very useful for better characterizing the variability of packet streams that are generated by such traffic sources, i.e., VoIP packet traffic sources. This exact expression of the variance-time curve is then compared with an approximated expression derived when considering the $\mathrm{ON} / \mathrm{OFF} /$ Exponential sources as a stationary fluid source.

\section{ON/OFF ExPONENTIAL SOURCE MOdeL}

We consider a packet stream (Fig. 1) generated by a single ON/OFF traffic source with strictly alternating ON- and OFFperiods. The OFF-periods are exponentially distributed with mean $\frac{1}{\beta}$, and during the ON-periods, the source transmits a packetized message whose size is also exponentially distributed. Hence, the number of packets $(W)$ transmitted during ON times is geometrically distributed, and thus the packet stream due to a single source is a renewal process. Assuming

Manuscript received February 12, 2007. The associate editor coordinating the review of this letter and approving it for publication was Prof. Maode Ma.

G. Y. Lazarou is with the New York City Transit (email: glaz@ieee.org).

V. S. Frost is with the University of Kansas (email: frost@ittc.ku.edu).

Digital Object Identifier 10.1109/LCOMM.2007.070233.

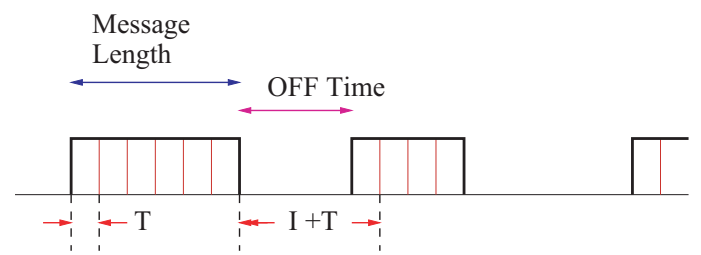

Fig. 1. Packet stream generated by a single ON/OFF source.

that the size of all packets transmitted is fixed, the probability density function (pdf) for the packet interarrival times is given by [1]

$$
f(x)=p \delta(x-T)+(1-p) \beta e^{-\beta(x-T)} u(x-T)
$$

where $u(t)$ is the unit step function, $T$ the packet transmission time, and $p(0<p<1)$ the probability that the next interarrival time ${ }^{1}$ is $T$. The interarrival time is measured from the last arrival bit of the $(n-1)^{s t}$ packet to the last arrival bit of the $n^{t h}$ packet. Define $E[W]$ as the mean number of packets transmitted during the $\mathrm{ON}$ periods, then

$$
p=\frac{E[W]-1}{E[W]} .
$$

That is, the interarrival times $X_{1}, X_{2}, \ldots, X_{n}$ are of length $T$ with probability $p$ and of length $I+T$ with probability $1-p$, where $I$ is the exponentially distributed random length of the OFF periods. The mean packet arrival rate is therefore

$$
\lambda=\frac{1}{E[X]}=\frac{1}{\int_{0}^{\infty} x f(x) d x}=\frac{\beta}{(1-p)+\beta T} .
$$

Let $\alpha^{-1}$ to be the mean ON time. Then $\alpha^{-1}=E[W] T$, and since $E[W]=\frac{1}{1-p}$, we have that $\alpha T=1-p$.

\section{Deriving the Variance-Time Curve}

Assuming an arbitrary origin, the renewal process $\{N(t), t \geq 0\}$ is stationary [3], where $N(t)$ denotes the number of packet arrivals in an interval of length $t$. The variance of arrival counts over a time interval of length $t$, or variance-time curve, is

$$
\begin{aligned}
\operatorname{Var}[N(t)] & =E\left[N^{2}(t)\right]-(E[N(t)])^{2} \\
& =E[N(t)\{N(t)+1\}]-E[N(t)]-(E[N(t)])^{2} \\
& =\Psi(t)-\lambda t-(\lambda t)^{2}
\end{aligned}
$$

where the challenge here is to obtain

$$
\Psi(t)=E[N(t)\{N(t)+1\}] .
$$

\footnotetext{
${ }^{1}$ We assume that packets from the same message are transmitted back to back without any inter-idle time.
} 
It is known from [4] that on taking the Laplace transform of $\Psi(t)$ we get

$$
\Psi^{*}(s)=\mathcal{L}[\Psi(t)]=\frac{2 \lambda}{s^{2}\left\{1-f^{*}(s)\right\}} \quad \text { for } \operatorname{Re}[s]>0
$$

where

$$
\begin{aligned}
f^{*}(s) & =\mathcal{L}[f(x)]=\int_{0}^{\infty} f(x) e^{-s x} d x \\
& =\left[p+(1-p) \frac{\beta}{s+\beta}\right] e^{-s T}
\end{aligned}
$$

is the Laplace transform of $f(x)$. Hence,

$$
\Psi^{*}(s)=\frac{2 \lambda}{s^{2}\left\{1-\left[p+(1-p) \frac{\beta}{s+\beta}\right] e^{-s T}\right\}}=\frac{\varphi^{*}(s)}{s^{2}}
$$

by letting

$$
\varphi^{*}(s)=\frac{2 \lambda}{1-\left[p+(1-p) \frac{\beta}{s+\beta}\right] e^{-s T}} .
$$

The next step in getting $\Psi(t)$ is to compute the inverse Laplace transform of $\Psi^{*}(s)$

$$
\Psi(t)=\mathcal{L}^{-1}\left[\Psi^{*}(s)\right]=\int_{c-j \infty}^{c+j \infty} \Psi^{*}(s) e^{s t} d s
$$

where $R e[s]=c>0$ being chosen so that all singularities of $\Psi^{*}(s)$ lie to the left of the line of integration.

Now, let $s=a+j b$ where $a=\operatorname{Re}[s]$ and $b=\operatorname{Im}[s]$. Then

$$
\begin{aligned}
\left|f^{*}(s)\right| & =\left|\left[p+(1-p) \frac{\beta}{s+\beta}\right] e^{-s T}\right| \\
& =\left|\left[p+(1-p) \frac{\beta}{a+j b+\beta}\right] e^{-(a+j b) T}\right| \\
& =\left|p+(1-p) \frac{\beta}{a+\beta+j b}\right|\left|e^{-(a+j b) T}\right| \\
& =\left|\frac{p a+p \beta+j p b+\beta-p \beta}{a+\beta+j b}\right| e^{-a T} \\
& =\left|\frac{p a+\beta+j p b}{a+\beta+j b}\right| e^{-a T} .
\end{aligned}
$$

Clearly, $e^{-a T}<1 \quad \forall a=R e[s]>0$ and

$$
\left|\frac{p a+\beta+j p b}{a+\beta+j b}\right|=\sqrt{\frac{(p a+\beta)^{2}+(p b)^{2}}{(a+\beta)^{2}+b^{2}}}<1
$$

for

$$
\begin{aligned}
& \forall 0<p<1, \beta>0, \\
& \forall a=\operatorname{Re}[s]>0, \text { and } \\
& \forall b=\operatorname{Im}[s],
\end{aligned}
$$

making

$$
\left|f^{*}(s)\right|<1 \quad \text { for } \operatorname{Re}[s]>0 .
$$

Thus, for $R e[s]>0$

$$
\varphi^{*}(s)=\frac{2 \lambda}{1-f^{*}(s)}
$$

is a geometric series ${ }^{2}$. Therefore, on the line of integration $\operatorname{Re}[s]=c>0$ in (9) $\left|f^{*}(s)\right|<1$ and the function $\varphi^{*}(s)$ is equal to

$$
\sum_{n=0}^{\infty} 2 \lambda\left[f^{*}(s)\right]^{n}=2 \lambda \sum_{n=0}^{\infty}\left[p+(1-p) \frac{\beta}{s+\beta}\right]^{n} e^{-s n T} .
$$

Upon using the Binomial Theorem ${ }^{3}$ in (11) we get

$$
\begin{aligned}
\varphi^{*}(s)= & 2 \lambda \sum_{n=0}^{\infty} \sum_{z=0}^{n}\left(\begin{array}{l}
n \\
z
\end{array}\right) p^{n-z}\left[(1-p) \frac{\beta}{s+\beta}\right]^{z} e^{-s n T} \\
= & 2 \lambda \sum_{n=0}^{\infty} p^{n} e^{-s n T}+ \\
& 2 \lambda \sum_{n=1}^{\infty} \sum_{z=1}^{n}\left(\begin{array}{l}
n \\
z
\end{array}\right) p^{n-z}(1-p)^{z} \beta^{z} \frac{e^{-s n T}}{(s+\beta)^{z}} .
\end{aligned}
$$

Letting

$$
\varphi_{1}^{*}(s)=2 \lambda \sum_{n=0}^{\infty} p^{n} e^{-s n T}
$$

and

$$
\varphi_{2}^{*}(s)=2 \lambda \sum_{n=1}^{\infty} \sum_{z=1}^{n}\left(\begin{array}{l}
n \\
z
\end{array}\right) p^{n-z}(1-p)^{z} \beta^{z} \frac{e^{-s n T}}{(s+\beta)^{z}},
$$

then $\varphi^{*}(s)=\varphi_{1}^{*}(s)+\varphi_{2}^{*}(s)$ and

$$
\Psi^{*}(s)=\frac{\varphi^{*}(s)}{s^{2}}=\frac{\varphi_{1}^{*}(s)}{s^{2}}+\frac{\varphi_{2}^{*}(s)}{s^{2}}=\Psi_{1}^{*}(s)+\Psi_{2}^{*}(s) .
$$

Obviously, $\Psi(t)=\Psi_{1}(t)+\Psi_{2}(t)$. Using the Laplace transform pair [5]

$$
\frac{e^{-a s}}{s^{2}} \stackrel{\mathcal{L}}{\Longleftrightarrow}(t-a) u(t-a) \quad a \geq 0
$$

we easily obtain

$$
\Psi_{1}(t)=2 \lambda \sum_{n=0}^{\infty} p^{n}(t-n T) u(t-n T) .
$$

Similarly, by using the Laplace transform pair

$$
\begin{aligned}
& \frac{e^{-s n T}}{s^{2}(s+a)^{z}} \stackrel{\mathcal{L}}{\Longleftrightarrow} \quad \frac{1}{a^{z+1}}\{a(t-n T) G[a(t-n T), z]- \\
&z G[a(t-n T), z+1]\} u(t-n T)
\end{aligned}
$$

for $a, n T \geq 0$ and $z=1,2, \ldots$, where

$$
G(x, y)=\frac{1}{\Gamma(y)} \int_{0}^{x} t^{y-1} e^{-t} d t \quad y>0, \quad x>0
$$

is the incomplete Gamma ${ }^{4}$ function, we get

$$
\begin{aligned}
\Psi_{2}(t)= & 2 \lambda \sum_{n=1}^{\infty} \sum_{z=1}^{n}\left(\begin{array}{l}
n \\
z
\end{array}\right) \frac{p^{n-z}(1-p)^{z}}{\beta}\{\beta(t-n T) \times \\
& G[\beta(t-n T), z]-z G[\beta(t-n T), z+1]\} u(t-n T) .
\end{aligned}
$$

A slightly different form of $\Psi_{2}(t)$ is presented in the Appendix, as well as a new mathematical identity based on the incomplete Gamma function derived by comparing the two forms of $\Psi_{2}(t)$.

\footnotetext{
${ }^{2}$ Geometric Series: $\sum_{n=0}^{\infty} \alpha x^{n}=\frac{\alpha}{1-x}$ for $|x|<1$.

${ }^{3}$ Binomial Theorem: $(a+x)^{n}=\sum_{z=0}^{n}\left(\begin{array}{l}n \\ z\end{array}\right) a^{n-z} x^{z}=a^{n}+$ $\left(\begin{array}{c}n \\ 1\end{array}\right) a^{n-1} x+\left(\begin{array}{c}n \\ 2\end{array}\right) x^{2}+\cdots+x^{n}$.

${ }^{4}$ Gamma Function: $\Gamma(y)=\int_{0}^{\infty} t^{y-1} e^{-t} d t \quad y>0$.
} 
Putting everything together, we then obtain the variancetime curve as:

$$
\begin{aligned}
\operatorname{Var}[N(t)] & =\Psi_{1}(t)+\Psi_{2}(t)-\lambda t-(\lambda t)^{2} \\
& =2 \lambda \sum_{n=0}^{\infty} p^{n}(t-n T) u(t-n T)+2 \lambda \sum_{n=1}^{\infty} \sum_{z=1}^{n}\left(\begin{array}{l}
n \\
z
\end{array}\right) \\
& \times \frac{p^{n-z}(1-p)^{z}}{\beta}\{\beta(t-n T) G[\beta(t-n T), z] \\
& -z G[\beta(t-n T), z+1]\} u(t-n T)-\lambda t-(\lambda t)^{2} .
\end{aligned}
$$

Suppose now an aggregated packet stream is generated by the superposition of $M$ independent but not necessarily identical ON/OFF/Exponential sources. Assuming again stationarity, then the index of dispersion for counts is given by

$$
I D C(t)=\frac{\sum_{i=1}^{M} \operatorname{Var}\left[N_{i}(t)\right]}{\sum_{i=1}^{M} \lambda_{i} t}
$$

where $\lambda_{i}$ is the mean packet arrival rate and $N_{i}(t)$ is the number of packet arrivals in the interval $(0, t]$ from the $i^{t h}$ source. In case that the sources are also identical, then the IDC for the aggregated packet stream is identical to that of a single source.

\section{Comparison with the Fluid Source Model}

Considering the ON/OFF/Exponential traffic source as a stationary fluid source with a constant transmission rate $\nu=\frac{1}{T}$ during the ON-periods, it can be described by a two-state Markov process. Letting $\rho=\alpha+\beta$, we then have from [6], [7] the approximate ${ }^{5}$ variance-time curve as

$$
\tilde{V} \operatorname{ar}[N(t)]=\frac{2(1-p) \lambda^{3}}{\beta^{2}}\left[t-\frac{1}{\rho}\left(1-e^{-\rho t}\right)\right] .
$$

One way to see that $\tilde{V} \operatorname{ar}[N(t)]$ is an approximate of $\operatorname{Var}[N(t)]$ given by (15) is by checking whether in the limit the index of dispersion for counts is equal with the squared coefficient of variation of the interarrival times ${ }^{6}, \mathcal{C}^{2}(X)$ : $\lim _{t \rightarrow \infty} I \tilde{D} C(t)=\frac{2}{1+p} \mathcal{C}^{2}(X)$. From this, the following is easily obtained:

$$
\lim _{t \rightarrow \infty} \frac{\tilde{V} \operatorname{Var}[N(t)]}{\operatorname{Var}[N(t)]}=\frac{2}{1+p} .
$$

Clearly, as the mean number of packets transmitted during the ON periods $(E[W])$ increases, and thus $p \rightarrow 1, \tilde{V}$ ar $[N(t)] \rightarrow$ $\operatorname{Var}[N(t)]$ for large enough $t$. But as $E[W]$ decreases and thus $p \rightarrow 0, \operatorname{Var}[N(t)] \rightarrow 2 \operatorname{Var}[N(t)]$ for large enough $t$. This shows that under low and medium loads, network performance obtained by using fluid analysis is over-estimated.

\footnotetext{
${ }^{5}$ During the ON-periods the burst of consecutive packets generated by the source is considered as a continuous fluid. Denote $I(t)=$

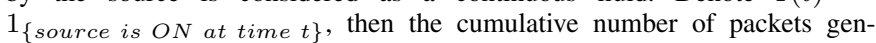
erated by the source in time interval $(0, t]$ is approximately $\tilde{N}(t)=$ $\int_{0}^{t} \nu I(t) d s$.

${ }^{6}$ Since $N(t)$ is a renewal process, then $\lim _{t \rightarrow \infty} I D C(t)=$ $\lim _{t \rightarrow \infty}(\operatorname{Var}[N(t)] / E[N(t)])=\mathcal{C}^{2}(X)=\operatorname{Var}[X] /(E[X])^{2}=\lambda^{2}(1-$ $\left.p^{2}\right) / \beta^{2}$.
}

\section{CONCLUSION}

We derived an exact expression of the variance-time curve using point processes analysis for packet streams generated by exponentially distributed ON/OFF network traffic sources. In addition, we showed that the fluid analysis over-estimates the variance-time curve under low or medium load conditions. Finally, our analysis generated a new mathematical identity based on the incomplete Gamma function.

\section{APPENDIX}

Let $\xi_{2}^{*}(s)=\frac{\varphi_{2}^{*}(s)}{s}$, so that $\Psi_{2}^{*}(s)=\frac{\varphi_{2}^{*}(s)}{s^{2}}=\frac{\xi_{2}^{*}(s)}{s}$ and

$$
\xi_{2}^{*}(s)=2 \lambda \sum_{n=1}^{\infty} \sum_{z=1}^{n}\left(\begin{array}{l}
n \\
z
\end{array}\right) p^{n-z}(1-p)^{z} \beta^{z} \frac{e^{-s n T}}{s(s+\beta)^{z}} .
$$

Applying the following Laplace transform pair [5]

$$
\frac{e^{-s n T}}{s(s+a)^{z}} \stackrel{\mathcal{L}}{\Longleftrightarrow} \frac{1}{a^{z}}\left\{1-e^{-a(t-n T)} \sum_{m=0}^{z-1} \frac{[a(t-n T)]^{m}}{m !}\right\} u(t-n T)
$$

for $a, n T \geq 0$ and $z=1,2, \ldots$, we get

$$
\begin{aligned}
\xi(t)= & 2 \lambda \sum_{n=1}^{\infty} \sum_{z=1}^{n}\left(\begin{array}{l}
n \\
z
\end{array}\right) p^{n-z}(1-p)^{z} \times \\
& \left\{1-e^{-\beta(t-n T)} \sum_{m=0}^{z-1} \frac{[\beta(t-n T)]^{m}}{m !}\right\} u(t-n T) .
\end{aligned}
$$

From this, an alternative form of $\Psi_{2}(t)$ is obtained as follows,

$$
\begin{aligned}
\Psi_{2}(t)= & \int_{0}^{t} \xi(x) d x=2 \lambda \sum_{n=1}^{\infty} \sum_{z=1}^{n}\left(\begin{array}{l}
n \\
z
\end{array}\right) \frac{p^{n-z}(1-p)^{z}}{\beta} \times \\
& \left\{\beta(t-n T)-\sum_{m=0}^{z-1} G[\beta(t-n T), m]\right\} u(t-n T) .
\end{aligned}
$$

Comparing the two different forms of $\Psi_{2}(t)$ shown in (15) and (18) we easily obtain the following identity

$\sum_{m=1}^{z} G(x, m)=x[1-G(x, z)]+z G(x, z+1) \quad z=1,2,3, \ldots$

To the best of authors' knowledge the above identity has never been published before.

\section{REFERENCES}

[1] K. Sriram and W. Whitt, "Characterizing superposition arrival processes in packet multiplexers for voice and data," IEEE J. Sel. Areas Commun., vol. 4, no. 6, pp. 833-846, Sept.1986.

[2] H. Heffes and D. M. Lucantoni, "A Markov modulated characterization of packetized voice and data traffic and related statistical multiplexer performance," IEEE J. Sel. Areas Commun., vol. 4, no. 6, pp. 856-868, Sept. 1986.

[3] D. R. Cox and P. A. Lewis, The Statistical Analysis of Series of Events. London: Methuen; New York: J. Wiley \& Sons, 1966.

[4] D. R. Cox, Renewal Theory. London: Methuen; New York: J. Wiley \& Sons, 1962.

[5] Paul A. McCollum and Buck F. Brown, Laplace Transform Tables And Theorems, New York: Holt, Rinehart and Winston, 1965.

[6] L.-S. Chou and C.-S. Chang, "Experiments of the theory of effective bandwidth for Markov sources and video traces," in Proc. IEEE INFO COM 1996, pp. 497-504.

[7] J. Roberts, U. Mocci, and J. Virtamo, Broadband network teletraffic: performance evaluation and design of broadband multiservice networks; final report of action COST 242, Springer, 1996. 